\title{
Serum Urate and Incidence of Kidney Disease Among Veterans with Gout
}

\author{
Eswar Krishnan, Kasem S. Akhras, Hari Sharma, Maryna Marynchenko, Eric Wu, \\ Rima H. Tawk, Jinan Liu, and Lizheng Shi
}

\begin{abstract}
Objective. To study the association between serum urate level (sUA) and the risk of incident kidney disease among US veterans with gouty arthritis.

Methods. From 2002 through 2011 adult male patients with gout who were free of kidney disease were identified in the data from the Veterans Administration VISN 16 database and were followed until incidence of kidney disease, death, or the last available observation. Accumulated hazard curves for time to kidney disease were estimated for patients with average sUA levels $>7 \mathrm{mg} / \mathrm{dl}$ (high) versus $\leq 7 \mathrm{mg} / \mathrm{dl}$ (low) based on Kaplan-Meier analyses; and statistical comparison was conducted using a log-rank test. A Cox proportional hazard model with time-varying covariates was used to estimate the unadjusted and adjusted hazard ratios for kidney disease.

Results. Eligible patients $(\mathrm{n}=2116)$ were mostly white $(53 \%)$, with average age 62.6 years, mean body mass index $31.2 \mathrm{~kg} / \mathrm{m}^{2}$, and high baseline prevalence of hypertension (93\%), hyperlipidemia $(67 \%)$, and diabetes $(20 \%)$. Mean followup time was 6.5 years. The estimated rates of all incident kidney disease in the overall low versus high sUA groups were $2 \%$ versus $4 \%$ at Year 1, 3\% versus $6 \%$ at Year 2, and 5\% versus 9\% at Year 3, respectively $(\mathrm{p}<0.0001)$. After adjustment, high sUA continued to predict a significantly higher risk of kidney disease development (HR 1.43, 95\% CI $1.20-1.70)$.

Conclusion. Male veterans with gout and sUA levels $>7 \mathrm{mg} / \mathrm{dl}$ had an increased incidence of kidney disease. (First Release May 15 2013; J Rheumatol 2013;40:1166-72; doi:10.3899/jrheum.121061)
\end{abstract}

Key Indexing Terms:

SERUM URATE URIC ACID GOUT KIDNEY DISEASE HYPERURICEMIA

Gout is by far the most common form of inflammatory arthritis, characterized by elevated levels of serum urate (sUA), which crystallizes and is deposited in joints, tendons, and surrounding tissues ${ }^{1}$. Based on data from the latest US National Health and Nutrition Examination Survey, gout affected about 7.5 million US adults in 2009-2010². Patients with gout frequently experience other medical problems, including metabolic syndrome, obesity, diabetes, hyper-

From Stanford University, Stanford, California; Takeda Pharmaceuticals International Inc., Deerfield, Illinois; Analysis Group Inc., Boston, Massachusetts; Tulane University, New Orleans, Louisiana; and Southeast Louisiana Veterans Health Care System, New Orleans, Louisiana, USA.

Supported by Takeda Pharmaceuticals International Inc. Dr. Krishnan has served as a consultant to Takeda Pharmaceuticals Inc., URL Pharmaceuticals Inc., Metabolex Inc., and UCB Pharmaceuticals Inc., and has received grant support from URL, ARDEA biosciences, and Takeda. R.H. Tawk is supported by the University of Illinois at Chicago/Takeda Fellowship.

E. Krishnan, MD, Stanford University; K.S. Akhras, PharmD, Takeda Pharmaceuticals International Inc.; H. Sharma, BS; M. Marynchenko, MBA; E. Wu, PhD, Analysis Group Inc.; R.H. Tawk, PhD, Takeda Pharmaceuticals International Inc.; J. Liu, PhD; L. Shi, PhD, Tulane University and Southeast Louisiana Veterans Health Care System.

Address correspondence to M. Marynchenko, Analysis Group Inc., 111 Huntington Avenue, Tenth Floor, Boston, MA 02199, USA.

E-mail: mmarynchenko@analysisgroup.com

Full Release Article. For details see Reprints/Permissions at jrheum.org Accepted for publication March 20, 2013. tension, and other cardiovascular diseases ${ }^{3,4,5}$. Additionally, between $30 \%$ and $60 \%$ of patients with gout exhibit a decrease in renal function over time ${ }^{6}$. In a recent study ${ }^{7}$ of the prevalence of chronic kidney disease, about 39\% of patients with gout had chronic kidney disease as defined by estimated glomerular filtration rate $<90 \mathrm{ml} / \mathrm{min} / 1.73 \mathrm{~m}^{2}$.

Hyperuricemia, or an abnormally high sUA concentration, has a substantial prevalence of $21 \%$ in the US and is a primary risk factor for the development of gout ${ }^{8}$. Recent reports indicate that hyperuricemia itself may contribute to the pathogenesis of certain gout-related comorbidities, including kidney disease ${ }^{6,9}$. Findings from animal studies have suggested several potential mechanisms by which elevated sUA levels could exacerbate renal impairment ${ }^{6,10,11}$; for example, sUA may play a key role in the development of renal microvascular disease, inflammation, and activation of the renin-angiotensin and cyclooxygenase-2 systems, events that in turn aggravate kidney disease and cardiovascular conditions ${ }^{6}$.

However, epidemiologic research has yielded conflicting evidence regarding the causal linkage between sUA concentration and the incidence and progression of chronic kidney disease $^{12}$. Studies have identified high levels of uric acid as a significant predictor of the development of kidney disease ${ }^{13,14,15,16,17,18,19,20}$. One prospective study conducted

Personal non-commercial use only. The Journal of Rheumatology Copyright (C) 2013. All rights reserved. 
among 21,457 seemingly healthy subjects found that the odds of new-onset kidney disease increased 1.74-fold with slightly elevated sUA ( 7.0 to $8.9 \mathrm{mg} / \mathrm{dl}$ ) and 3.12 -fold with elevated sUA $(\geq 9.0 \mathrm{mg} / \mathrm{dl})$ relative to normal sUA $(<7.0$ $\mathrm{mg} / \mathrm{dl})^{14}$. Conversely, other reports have not supported the hypothesis that sUA contributes to chronic kidney disease $^{21,22,23,24}$, including a cohort study of 5808 elderly participants in which sUA showed a significant association with prevalent but not incident kidney disease ${ }^{21}$.

The relationship between sUA concentration and kidney disease requires further clarification, and has not been studied extensively within gout-specific populations. Risks of hyperuricemia and renal impairment are high among patients with gout; thus, it is important to understand the implications of sUA control in this disease area. Using a retrospective cohort design, we estimated the effect of sUA levels on the risk of developing new-onset kidney disease in male US veterans diagnosed with gout.

\section{MATERIALS AND METHODS}

Data source. The database analysis was performed using electronic medical records from the South Central Veterans' Affairs Health Care Network (VISN 16) data warehouse. The VISN 16 data warehouse is an integrated, de-identified, individual-level database that includes records for more than 445,000 veterans located in Arkansas, Louisiana, Mississippi, Oklahoma, and parts of Alabama, Florida, Missouri, and Texas. The health care network represents 10 medical centers and 40 community-based outpatient clinics. The database contains demographic data, inpatient and outpatient records, pharmacy prescriptions, laboratory results, vital sign data (height, body weight), and mortality information (date of death) for each patient treated within the network. The data are updated monthly and maintained by the VISN 16 Information Technology Development Group. Data covering the period January 1, 2002, to January 1, 2011, were used for our study.

Sample selection. Patients were eligible for study if they had at least 2 recorded sUA measurements between June 30, 2002, and January 1, 2011, and were continuously enrolled in the database for a minimum of 6 months before and 12 months after their first sUA measurement during this period. The index date for each patient was defined as the date of the first sUA measurement. Patients without at least 2 sUA values recorded during their continuous eligibility period were excluded.

The study sample was further restricted to patients aged $\geq 18$ years who received at least 2 separate diagnoses of gout [International Classification of Disease-9 Clinical Modification (ICD-9-CM): 274.xx] on different dates. Because veterans enrolled in the VISN 16 database are predominantly male, the study excluded female subjects. Patients were excluded if they received any diagnoses for other inflammatory diseases including rheumatoid arthritis (ICD-9: 714), diffuse diseases of connective tissue such as lupus, scleroderma, etc. (ICD-9-CM: 710), vasculitis (ICD-9-CM: 446), psoriatic arthritis (ICD-9-CM: 696.0), autoimmune disease (ICD-9-CM: 283.0, 580-583, 242.0, 340, 358.0, 130.3, 422.0, 422.9, 429.0, 390-398), pseudogout (ICD-9-CM: 712.2, 727.82, 275.4), and other inflammatory arthritis (ICD-9-CM: 711.1, 711.3, 712, 713.1, 720). Finally, patients were excluded if they had a history of kidney disease prior to the index date, identified by recorded diagnosis codes (ICD-9-CM: 580-588, 250.4, 590, 593, 791.0) and/or procedure codes for dialysis [Current Procedural Terminology (CPT): 90935, 90937, 90945, and 90947]. Even though the specific ICD-9-CM codes used to define kidney disease vary somewhat across studies, they have been widely used in the literature $24,25,26,27$.
Endpoint assessment. The endpoint assessed in our study was time to first diagnosis of kidney disease, starting from the index date and using the same ICD-9 codes as for the exclusion criterion above. All measures of sUA concentration and the covariates of interest during the post-index period were included in the analysis. Each patient's mean sUA level was estimated for each 6-month cycle starting from the index date until the end of continuous eligibility. Because sUA readings were not available for every cycle, linear extrapolation using adjacent sUA measurements was applied to obtain sUA levels for all cycles. These measures were then dichotomized as low $(\leq 7 \mathrm{mg} / \mathrm{dl})$ or high $(>7 \mathrm{mg} / \mathrm{dl})$ sUA within each 6-month interval. Additionally, for the purposes of descriptive analyses, the patient sample was divided into overall low or high sUA groups according to whether average sUA level over the entire study period was $\leq 7 \mathrm{mg} / \mathrm{dl}$ or $>7 \mathrm{mg} / \mathrm{dl}$, respectively; the average of sUA measurements was estimated using the average area under the curve (AUC) method. There is variation in the literature in the sUA concentration level used to define hyperuricemia. However, many studies define hyperuricemia in men as sUA $\geq 7$ $\mathrm{mg} / \mathrm{dl}^{9,14,28,29}$. For this study, sUA $>7 \mathrm{mg} / \mathrm{dl}$ was considered a high sUA.

All other predictors of kidney disease were measured using health records during the 6-month pre-index baseline period: age at the index date, year of index date, race (white or nonwhite), state of residence (Arkansas, Louisiana, Mississippi, Oklahoma, or Texas), body mass index (BMI), and baseline tobacco use, diabetes, hyperlipidemia, and hypertension. These factors were selected a priori based on the existing literature and data availability $16,30,31,32,33$.

Statistical analysis. Baseline characteristics assessed during the 6-month period prior to the index date were summarized for the overall study sample in terms of mean $\pm \mathrm{SD}$ or proportion.

In the unadjusted Kaplan-Meier analysis, time to first kidney disease diagnosis was compared between the overall low versus overall high sUA groups. Accumulated hazard curves for time to event were estimated for both sUA groups, and the statistical comparison was conducted using a log-rank test. The multivariate adjusted analysis used a Cox proportional hazard model to estimate the relative risk of kidney disease associated with high versus low sUA, with sUA category assessed during each 6-month cycle as a time-varying covariate. To address the correlation between different cycles for the same patient, a model-based, robust sandwich estimate was estimated for the covariance matrix resulting in robust standard errors for the parameters ${ }^{34}$. The model adjusted for the full set of demographics (age, race, region, year of index date) and baseline risk factors for kidney disease (BMI, hypertension, diabetes, smoking, and hyperlipidemia) measured in our study. The estimated association of sUA level and risk of kidney disease was expressed using a hazard ratio (HR) and $95 \% \mathrm{CI}$.

As additional exploratory analyses, we tested each baseline predictor in a univariate Cox proportional hazards model to determine whether it was associated with risk of incident kidney disease in the study population. The use of gout medications (allopurinol, colchicines, probenecid, and febuxostat) prior to kidney disease diagnosis date was descriptively summarized for the 2 sUA cohorts. In addition, in the main analysis, we required 2 gout diagnoses (ICD-9-CM codes) to identify patients with gout. To assess whether this definition of gout was robust, we conducted additional sensitivity analyses using 2 different gout definitions: at least 3 gout diagnoses; and at least 2 gout diagnoses and at least 1 gout medication.

To assess whether the initial kidney disease diagnosis was merely due to errors in coding or an exploratory diagnosis that was not confirmed later, we estimated the proportions of patients with subsequent kidney disease diagnosis within Years 1,2, and 3 following the initial kidney disease diagnosis. Additionally, because hypertension is an independent risk factor for kidney disease and was potentially not well managed during the study period, we conducted additional sensitivity analysis for the risk of kidney disease by using diagnosis of hypertension or the use of antihypertensive medications as time-varying risk factors in the Cox proportional hazards model.

Statistical significance was assessed at the 5\% level. Analyses were conducted using SAS version 9.2

Personal non-commercial use only. The Journal of Rheumatology Copyright @ 2013 . All rights reserved. 


\section{RESULTS}

A total of 2116 male veterans with gout and no recorded history of kidney disease were eligible for inclusion. Detailed sample counts from the selection process are provided in Figure 1. All study subjects had $\geq 2$ sUA measurements and were continuously eligible for a minimum of 12 months from the first sUA recording. On average, patients were followed for a total of $12.9( \pm 4.4) 6$-month cycles (roughly 6.5 years), and had $3.9( \pm 2.7)$ separate non-missing sUA values recorded during followup, including the initial baseline value. Based on average sUA level throughout the study period, 1204 patients $(56.9 \%)$ were categorized into the overall high sUA group and the remaining $912(43.1 \%)$ were included in the overall low sUA group.

Baseline characteristics are summarized in Table 1. Average age in the study population was 62.6 years $( \pm 11.6)$, and nonwhite subjects constituted $47 \%$ of the sample. The majority of veterans studied resided in Mississippi (55\%). Mean BMI was high at $31.2 \mathrm{~kg} / \mathrm{m}^{2}( \pm 6.8)$ and a majority of patients had a history of hypertension (93\%) and hyperlipidemia $(67 \%)$ during the 6-month pre-index period.

The unadjusted Kaplan-Meier analysis found that patients with overall high sUA levels had significantly higher rates of new onset of kidney disease over time than those with overall low sUA ( $p<0.0001)$. As shown by the accumulated hazard curves (Figure 2), the divergence in rates of kidney disease between the groups emerged immediately following the index date and was apparent throughout the study period. The estimated rates of incident kidney disease in the overall low versus high sUA groups were $2 \%$ versus $4 \%$ at Year 1,3\% versus $6 \%$ at Year 2, and $5 \%$ versus $9 \%$ at Year 3, respectively.

Multivariate regression-adjusted results were consistent with descriptive findings. High sUA levels significantly predicted a higher risk of new onset of kidney disease relative to low sUA levels (HR 1.43, 95\% CI 1.20-1.70) after controlling for baseline characteristics (Table 2).

Based on univariate Cox proportional hazard models, statistically significant predictors of new-onset kidney disease included age (HR 1.03, 95\% CI 1.02-1.04), baseline presence of diabetes (HR 1.68, 95\% CI 1.40-2.02), and hypertension (HR 2.13, 95\% CI 1.41-3.24). Use of gout medication was comparable between patients with overall high versus low sUA prior to diagnosis of kidney disease $(89.5 \%$ vs $90.1 \%$; $\mathrm{p}=0.6535)$.

Sensitivity analysis. Additional sensitivity analyses using different definitions to identify patients with gout confirmed the findings from our main analysis. Among patients with 2 gout diagnoses and at least 1 gout medication use, high sUA levels significantly predicted a higher risk of new onset of kidney disease relative to low sUA levels (HR 1.44, 95\% CI $1.20-1.72)$. The risk of new kidney disease was also higher for high versus low sUA levels (HR 1.49, 95\% CI $1.24-1.79)$ when the analysis was conducted only on patients with at least 3 gout diagnoses (Table 2).

The findings were consistent when hypertension diagnosis or the use of antihypertensive medications was used as a time-varying covariate. In all groups of patients, high sUA levels were associated with a significantly higher risk of new onset of kidney disease.

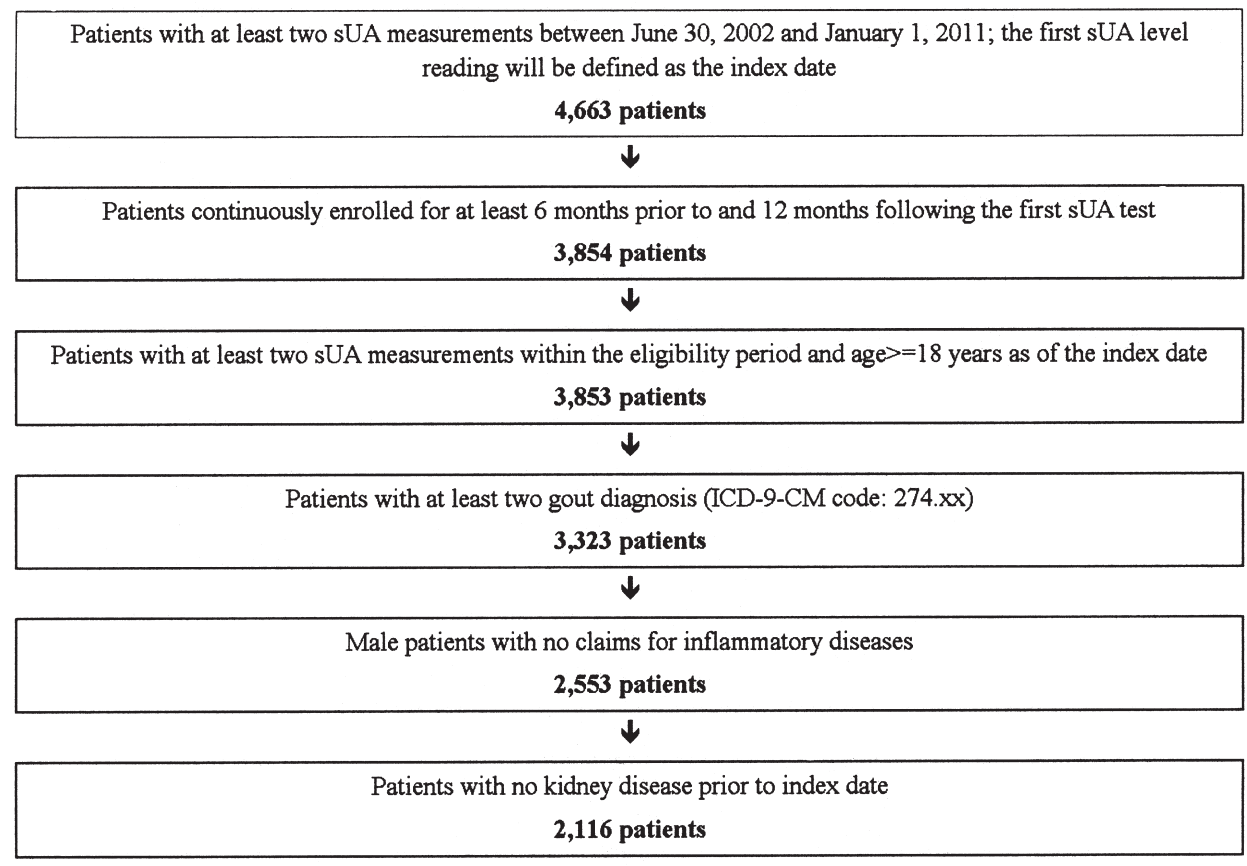

Figure 1. The selection process for study subjects. ICD: International Classification of Disease-9 Clinical Modification; sUA: serum urate level. 
Table 1. Patient characteristics. A patient is considered to have high serum urate (sUA) if the average area under the curve for sUA levels is $>7$ during the study period. Otherwise, the patient is considered to have low sUA. High versus low samples are used for survival analysis.

\begin{tabular}{|c|c|c|c|c|}
\hline \multirow[b]{2}{*}{ Characteristics } & \multicolumn{4}{|c|}{ Patients with No Kidney Disease Prior to Index Date } \\
\hline & $\begin{array}{l}\text { All Patients, } \\
\mathrm{n}=2116\end{array}$ & $\begin{array}{c}\text { High sUA, } \\
\mathrm{n}=1204\end{array}$ & $\begin{array}{l}\text { Low sUA, } \\
\mathrm{n}=912\end{array}$ & $\begin{array}{c}\mathrm{p}, \\
\text { High vs Low sUA }\end{array}$ \\
\hline \multicolumn{5}{|l|}{ Demographic } \\
\hline $\begin{array}{l}\text { Age at } 1 \text { st sUA test date, yrs; } \\
\text { mean } \pm \text { SD }\end{array}$ & $62.6 \pm 11.63$ & $61.1 \pm 11.86$ & $64.5 \pm 11.04$ & $<0.0001$ \\
\hline Race, $\mathrm{n}(\%)$ & & & & $<0.0001$ \\
\hline White & $1120(53)$ & $565(47)$ & $555(61)$ & \\
\hline Others & $996(47)$ & $639(53)$ & $357(39)$ & \\
\hline Region, $\mathrm{n}(\%)$ & & & & $<0.0001$ \\
\hline Arkansas & $341(16)$ & $159(13)$ & $182(20)$ & \\
\hline Louisiana & $368(17)$ & $219(18)$ & $149(16)$ & \\
\hline Mississippi & $1157(55)$ & $695(58)$ & $462(51)$ & \\
\hline Oklahoma & $223(11)$ & $116(10)$ & $107(12)$ & \\
\hline Texas & $27(1)$ & $15(1)$ & $12(1)$ & \\
\hline Body mass index, mean $\pm \mathrm{SD}$ & $31.2 \pm 6.79$ & $31.5 \pm 6.24$ & $30.9 \pm 7.45$ & 0.0250 \\
\hline \multicolumn{5}{|l|}{ Average no. of 6-month cycles } \\
\hline during the study period, mean \pm SD & $12.9 \pm 4.37$ & $12.6 \pm 4.46$ & $13.3 \pm 4.22$ & 0.0008 \\
\hline Average no. of sUA values, mean \pm SD & $3.9 \pm 2.68$ & $4.1 \pm 2.99$ & $3.6 \pm 2.17$ & 0.0001 \\
\hline Index year, $\mathrm{n}(\%)$ & & & & 0.3930 \\
\hline 2002 & $632(30)$ & $340(28)$ & $292(32)$ & \\
\hline 2003 & $576(27)$ & $326(27)$ & $250(27)$ & \\
\hline 2004 & $249(12)$ & $143(12)$ & $106(12)$ & \\
\hline 2005 & $209(10)$ & $121(10)$ & $88(10)$ & \\
\hline 2006 & $134(6)$ & $78(6)$ & $56(6)$ & \\
\hline 2007 & $116(5)$ & $70(6)$ & $46(5)$ & \\
\hline 2008 & $115(5)$ & $76(6)$ & $39(4)$ & \\
\hline 2009 & $85(4)$ & $50(4)$ & $35(4)$ & \\
\hline \multicolumn{5}{|l|}{ Comorbidities, n (\%) } \\
\hline Diabetes & $416(20)$ & $205(17)$ & $211(23)$ & $<0.0001$ \\
\hline Hyperlipidemia & $1,412(67)$ & $769(64)$ & $643(71)$ & 0.0010 \\
\hline Hypertension & $1,964(93)$ & $1,121(93)$ & $843(92)$ & 0.5530 \\
\hline Smoking & $173(8)$ & $116(10)$ & $57(6)$ & 0.0050 \\
\hline
\end{tabular}

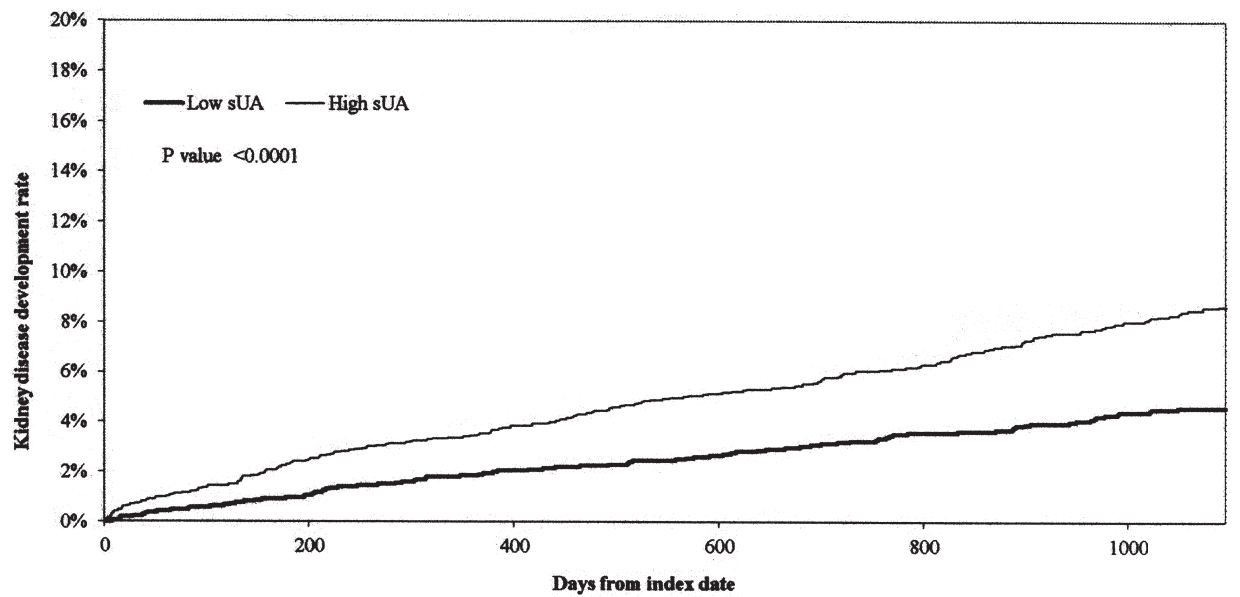

Figure 2. Accumulated hazard curve for time to the first diagnosis of kidney disease. Patients with high serum urate (sUA) have a significantly higher kidney disease diagnosis rate compared to those with low sUA. A patient is considered to have high sUA if average area under the curve for sUA levels was $>7$ during the study period. Time to kidney disease was measured from index date until kidney disease diagnosis, death, or the end of data collection, whichever came first. Patients with kidney disease prior to the index date were excluded. 
Table 2. Hazard ratios for development of kidney disease for patients with no kidney disease prior to index date. Cox proportional hazard model was estimated controlling for age, index year, region, body mass index, race, tobacco use - past or present, and baseline hyperlipidemia, hypertension, and diabetes. Serum urate level was used as a time-varying covariate.

\begin{tabular}{lcccc}
\hline $\begin{array}{l}\text { Identification of Patients with Gout } \\
\text { At least 2 gout diagnoses }\end{array}$ & $\mathrm{N}$ & Hazard Ratio & $\begin{array}{c}\text { Lower 95\% } \\
\text { Confidence Limit }\end{array}$ & $\begin{array}{c}\text { Upper 95\% } \\
\text { Confidence Limit }\end{array}$ \\
$\begin{array}{l}\text { At least 2 gout diagnoses and at } \\
\text { least 1 gout medication }\end{array}$ & 2116 & 1.43 & 1.20 & 1.70 \\
At least 3 gout diagnoses & 2006 & 1.44 & 1.20 & 1.72 \\
& 1879 & 1.49 & 1.24 & 1.79 \\
\hline
\end{tabular}

Among the 564 patients with kidney disease during the study period, 455 patients had at least 1 year of continuous followup after the initial kidney disease diagnosis. Of these 455 patients, $53.4 \%$ had another kidney disease diagnosis claim within the 1 -year period. Among the 372 patients with a new kidney disease diagnosis and at least 2 years of continuous followup, 51.9\% had another kidney disease diagnosis within 1 year, and $61.0 \%$ within 2 years following the initial diagnosis. Similarly, 270 patients had a new kidney disease diagnosis and had at least 3 years of continuous followup. Of these 270 patients, $50.0 \%$, 59.6\%, and $65.9 \%$ had another kidney disease diagnosis within 1 year, 2 years, and 3 years following the initial diagnosis.

\section{DISCUSSION}

Our retrospective cohort study evaluated the association between sUA levels and risk of new-onset kidney disease diagnoses among male US veterans with gout and no prior evidence of kidney disease. The burden of hyperuricemia was high among study subjects, with about $60 \%$ of patients demonstrating an average sUA level $>7 \mathrm{mg} / \mathrm{dl}$ during followup. Patients classified as having high sUA had a 9\% risk of being newly diagnosed with kidney disease by the end of Year 3, compared to a 5\% risk in the low sUA group $(\mathrm{p}<0.0001)$. In the longitudinal multivariate analysis, elevated sUA levels continued to predict a significantly higher risk of new kidney disease after adjustment for demographics and baseline health factors. Sensitivity analyses using different gout definitions or the use of hypertension diagnosis/antihypertensive medications as time-varying covariates confirmed this finding.

While it is difficult to compare the kidney disease development rates from our study to those from the general population because of differences in definitions, study design, and patient populations, the incidence of recognized chronic kidney disease in the US Medicare population aged 65 years and older was $4.3 \%$, but ranged between $0.62 \%$ and $0.71 \%$ for those between the ages of 45 and 64 years in $2008^{35}$. In our study, patients with low serum urate had an average age of 64.5 years, and the kidney disease development rate at Year 3 was $5 \%$.

The main results corroborate findings from earlier studies that detected a statistically significant association between sUA level and risks of kidney disease development and/or progression. The contributory role of sUA in kidney disease has been supported by several analyses of general community-based populations from the US ${ }^{14,16,17}$ and other countries ${ }^{15,18,19,20}$. The largest of these studies, conducted among 177,570 members of an integrated health system in California, found that sUA level in the highest quartile predicted a 2.14-fold increase in the hazard of developing endstage kidney disease relative to the lowest quartile, after adjustment for a comprehensive set of other potential confounders ${ }^{16}$. A few other studies have reported similar results within disease-specific populations. Elevated uric acid was associated with a significantly increased risk of chronic kidney disease in hypertensive women ${ }^{36}$ and was correlated with a more rapid decline in residual renal function among patients undergoing peritoneal dialysis ${ }^{37}$. The present analysis, which was conducted in a distinct patient population of male veterans diagnosed with gout, adds to the body of evidence that elevated sUA is an independent risk factor for new-onset kidney disease. Our findings suggest that sUA may have important clinical implications in terms of managing complications such as development of kidney disease in patients with gout, beyond its utility as an indicator for gout severity.

Not all epidemiologic studies have identified hyperuricemia as an independent predictor of kidney disease $21,22,23,24$. A community-based study of elderly individuals found that higher quintiles of sUA correlated significantly with prevalent kidney disease in a cross-sectional analysis, and predicted kidney disease progression based on decreases in glomerular filtration rate; however, baseline sUA (as a continuous variable) did not show any association with the development of new kidney disease $^{21}$. Reasons for the discrepancy between these studies and our results are unclear, but may be related to differences in patient population or other study features. Of note, our study assessed sUA level as a time-varying factor to account for updates in patients' sUA status after the index date; on average, patients in the study sample had 3.9 sUA measurements available during followup. Earlier studies instead evaluated initial sUA at baseline as a predictor of

Personal non-commercial use only. The Journal of Rheumatology Copyright (C) 2013. All rights reserved 
kidney disease development; use of baseline sUA may be more susceptible to misclassification bias toward the null if, for example, a nontrivial number of patients achieve reductions in sUA before the onset of renal impairment.

The identification of hyperuricemia as a modifiable risk factor raises the question of whether interventions aimed at sUA control have the potential to reduce the risk of kidney disease in patients with gout. In a recent posthoc analysis of a clinical trial evaluating hyperuricemic gout patients receiving febuxostat therapy for up to 5 years, a $1 \mathrm{mg} / \mathrm{dl}$ decrease in sUA was projected to yield an improvement of 1 $\mathrm{ml} / \mathrm{min}$ in estimated glomerular filtration rate ${ }^{38}$. To inform clinical management guidelines, additional evidence from clinical trials is needed to confirm the longterm efficacy of urate-lowering therapy in improving renal function among hyperuricemic gout patients.

Our study is subject to several limitations, including the general limitations of observational and retrospective analyses. Although every effort was made to control for confounding differences between patients with low versus high sUA, unobserved confounding factors may have led to bias that was not fully adjustable. As with other electronic medical record databases, the VISN 16 database may not capture all clinical activity of the selected patients. Additionally, all patients were enrolled in the Veterans Affairs network, which may reduce the representativeness of the study sample. The greater homogeneity of the patient sample reduces the potential for confounding but may limit the generalizability of the results to the overall US gout population. In addition, our study used a well-established cutoff point of sUA $>7 \mathrm{mg} / \mathrm{dl}$ to identify high sUA, although more recently, several studies used sUA $>6 \mathrm{mg} / \mathrm{dl}$ as a cutoff point for men. Finally, our observational study identified gout using diagnosis codes. Availability of other clinical measures could have added reliability to the identification of gout, but a recent study that assessed the validity of using ICD-9 codes to identify gout in the VA database suggested that this database can be used to identify gout-related visits with good accuracy ${ }^{39}$. Similarly, our study identified kidney disease on the basis of diagnosis and procedure codes rather than clinical laboratory measurements, because serum creatinine and glomerular filtration rate values were not available. It is difficult to know whether the identification of kidney disease using diagnosis and procedure codes underestimated or overestimated the actual presence of kidney disease, but this outcome definition was not expected to cause systematic differences between the cohorts. Our sensitivity analysis of patients with a new kidney disease diagnosis showed that the majority of these patients had another kidney disease diagnosis within a year following the initial diagnosis. The use of ICD-9 codes to identify kidney disease is common in the literature, even though the specific ICD-9 codes vary across studies ${ }^{24,25,26,27}$. To our knowledge, there are no studies validating the use of
ICD-9 diagnosis to identify kidney disease in the VA database, but some studies have validated the use of ICD-9 codes to identify patients with chronic kidney disease in Medicare claims ${ }^{40}$.

In this US Veterans Affairs population, gout patients with sUA $>7 \mathrm{mg} / \mathrm{dl}$ developed more kidney disease as defined using ICD-9-CM and CPT procedure codes than gout patients with sUA $<7 \mathrm{mg} / \mathrm{dl}$. Followup intervention studies are needed to assess the feasibility of preventing kidney disease in hyperuricemic gout patients through reductions in sUA levels. Further studies on the contribution of different sUA levels and treatments on the development of kidney disease among gout patients are warranted.

\section{ACKNOWLEDGMENT}

The authors thank Arielle Bensimon, Analysis Group Inc., for support in the preparation of this report; the US Department of Veterans Affairs VISN 16 data warehouse for the de-identified dataset, and the Southeast Louisiana Veterans Health Care system for additional resources.

\section{REFERENCES}

1. Crittenden DB, Pillinger MH. The year in gout $-2010-2011$. Bull NYU Hosp Jt Dis 2011;69:257-63.

2. Krishnan E. Reduced glomerular function and prevalence of gout: NHANES 2009-10. PLoS ONE 2012;7:e50046.

3. Annemans L, Spaepen E, Gaskin M, Bonnemaire M, Malier V, Gilbert T, et al. Gout in the UK and Germany: prevalence, comorbidities and management in general practice 2000-2005. Ann Rheum Dis 2008;67:960-6.

4. Terkeltaub R, Zelman D, Scavulli J, Perez-Ruiz F, Liote F. Gout Study Group: update on hyperuricemia and gout. Joint Bone Spine 2009;76:444-6.

5. Krishnan E. Gout and coronary artery disease: epidemiologic clues. Curr Rheumatol Rep 2008;10:249-55.

6. Kang DH, Nakagawa T. Uric acid and chronic renal disease: possible implication of hyperuricemia on progression of renal disease. Semin Nephrol 2005;25:43-9.

7. Fuldeore MJ, Riedel AA, Zarotsky V, Pandya BJ, Dabbous O, Krishnan E. Chronic kidney disease in gout in a managed care setting. BMC Nephrol 2011;12:36.

8. Zhu Y, Pandya BJ, Choi HK. Prevalence of gout and hyperuricemia in the US general population: The National Health and Nutrition Examination Survey 2007-2008. Arthritis Rheum 2011;63:3136-41.

9. Heinig M, Johnson RJ. Role of uric acid in hypertension, renal disease, and metabolic syndrome. Cleve Clin J Med 2006; 73:1059-64.

10. Ginsberg MH, Kozin F, O'Malley M, McCarty DJ. Release of platelet constituents by monosodium urate crystals. J Clin Invest 1977;60:999-1007.

11. Sánchez-Lozada LG, Tapia E, Santamaría J, Avila-Casado C, Soto $\mathrm{V}$, Nepomuceno T, et al. Mild hyperuricaemia induces vasoconstriction and maintains glomerular hypertension in normal and remnant kidney rats. Kidney Int 2005;67:237-47.

12. Feig DI. Uric acid: A novel mediator and marker of risk in chronic kidney disease? Curr Opin Nephrol Hypertens 2009;18:526-30.

13. Avram Z, Krishnan E. Hyperuricaemia - Where nephrology meets rheumatology. Rheumatology 2008;47:960-4.

14. Obermayr RP, Temml C, Gutjahr G, Knechtelsdorfer M, Oberbauer R, Klauser-Braun R. Elevated uric acid increases the risk for kidney disease. J Am Soc Nephrol 2008;19:2407-13.

15. Chen YC, Su CT, Wang ST, Lee HD, Lin SY. A preliminary Personal non-commercial use only. The Journal of Rheumatology Copyright $@$ $~ 2013$. All rights reserved 
investigation of the association between serum uric acid and impaired renal function. Chang Gung Med J 2009;32:66-71.

16. Hsu CY, Iribarren C, McCulloch CE, Darbinian J, Go AS. Risk factors for end-stage renal disease: 25-year follow-up. Arch Intern Med 2009;169:342-50.

17. Weiner DE, Tighiouart H, Elsayed EF, Griffith JL, Salem DN, Levey AS. Uric acid and incident kidney disease in the community. J Am Soc Nephrol 2008;19:1204-11.

18. Iseki K, Oshiro S, Tozawa M, Iseki C, Ikemiya Y, Takishita S. Significance of hyperuricemia on the early detection of renal failure in a cohort of screened subjects. Hypertens Res 2001;24:691-7.

19. Chen N, Wang W, Huang Y, Shen P, Pei D, Yu H, et al. Community-based study on CKD subjects and the associated risk factors. Nephrol Dial Transplant 2009;24:2117-23.

20. Bellomo G, Venanzi S, Verdura C, Saronio P, Esposito A, Timio M. Association of uric acid with change in kidney function in healthy normotensive individuals. Am J Kidney Dis 2010;56:264-72.

21. Chonchol M, Shlipak MG, Katz R, Sarnak MJ, Newman AB, Siscovick DS, et al. Relationship of uric acid with progression of kidney disease. Am J Kidney Dis 2007;50:239-47.

22. Sturm G, Kollerits B, Neyer U, Ritz E, Kronenberg F. Uric acid as a risk factor for progression of non-diabetic chronic kidney disease? The Mild to Moderate Kidney Disease (MMKD) Study. Exp Gerontol 2008;43:347-52.

23. See LC, Kuo CF, Chuang FH, Li HY, Chen YM, Chen HW, et al. Serum uric acid is independently associated with metabolic syndrome in subjects with and without a low estimated glomerular filtration rate. J Rheumatol 2009;36:1691-8.

24. Madero M, Sarnak MJ, Wang X, Greene T, Beck GJ, Kusek JW, et al. Uric acid and long-term outcomes in CKD. Am J Kidney Dis 2009;53:796-803.

25. Griffin MR, Yared A, Ray WA. Nonsteroidal antiinflammatory drugs and acute renal failure in elderly persons. Am J Epidemiol 2000;151:488-96.

26. Meliker JR, Wahl RL, Cameron LL, Nriagu JO. Arsenic in drinking water and cerebrovascular disease, diabetes mellitus, and kidney disease in Michigan: a standardised mortality ratio analysis. Environ Health 2007;6:4.

27. Van Staa, TP, Travis S, Leufkens HG, Logan RF. 5-aminosalicylic acids and the risk of renal disease: a large British epidemiologic study. Gastroenterology 2004;126:1733-9.
28. Sonoda H, Takase H, Dohi Y, Kimura G. Uric acid levels predict future development of chronic kidney disease. Am J Nephrol 2011;33:352-7.

29. Iseki K, Ikemiya Y, Inoue T, Iseki C, Kinjo K, Takishita S. Significance of hyperuricemia as a risk factor for developing ESRD in a screened cohort. Am J Kidney Dis 2004;44:642-50.

30. Hsu CY, Ordonez JD, Chertow GM, Fan D, McCulloch CE, Go AS The risk of acute renal failure in patients with chronic kidney disease. Kidney Int 2008;74:101-7.

31. Reynolds K, Gu D, Muntner P, Chen J, Wu X, Yau CL, et al. Body mass index and risk of ESRD in China. Am J Kidney Dis 2007;50:754-64.

32. Hunsicker LG, Adler S, Caggiula A, England BK, Greene T, Kusek JW, et al. Predictors of the progression of renal disease in the Modification of Diet in Renal Disease Study. Kidney Int 1997;51:1908-19.

33. Klahr S, Schreiner G, Ichikawa I. The progression of renal disease. N Engl J Med 1988;318:1657-66.

34. Lin DY, Wei LJ. The robust inference for the Cox proportional hazards model. J Am Stat Assoc 1989;84:1074-8.

35. US Renal Data System. USRDS 2010 annual data report: Atlas of chronic kidney disease and end-stage renal disease in the United States. Bethesda: National Institutes of Health, National Institute of Diabetes and Digestive and Kidney Diseases; 2010:2.

36. Borges RL, Hirota AH, Quinto BM, Ribeiro AB, Zanella MT, Batista MC. Uric acid as a marker for renal dysfunction in hypertensive women on diuretic and nondiuretic therapy. J Clin Hypertens 2009;11:253-9.

37. Park JT, Kim DK, Chang TI, Kim HW, Chang JH, Park SY, et al. Uric acid is associated with the rate of residual renal function decline in peritoneal dialysis patients. Nephrol Dial Transplant 2009;24:3520-5.

38. Whelton A, Macdonald PA, Zhao L, Hunt B, Gunawardhana L. Renal function in gout: long-term treatment effects of febuxostat. J Clin Rheumatol 2011;17:7-13.

39. Singh JA. Accuracy of Veterans Affairs database for gout-related health care utilization [abstract]. Arthritis Rheum 2012:63 Suppl:S771.

40. Winkelmayer WC, Schneeweiss S, Mogun H, Patrick AR, Avorn J, Solomon DH. Identification of individuals with CKD from Medicare claims data: a validation study. Am J Kidney Dis $2005 ; 46: 225-32$. 